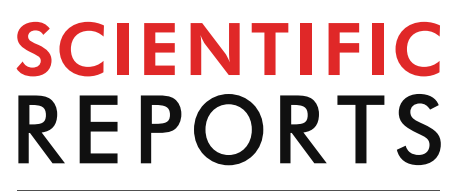

natureresearch

Check for updates

\title{
OPEN Microenvironment-triggered dual-activation of a photosensitizer- fluorophore conjugate for tumor specific imaging and photodynamic therapy
}

\author{
Chang Wang ${ }^{1}$, Shengdan Wang ${ }^{1}$, Yuan Wang ${ }^{1}$, Honghai Wu ${ }^{1}$, Kun Bao ${ }^{2}$, Rong Sheng ${ }^{1 \bowtie}$ \& \\ Xin $\mathrm{Li}^{1 \bowtie}$
}

Photodynamic therapy is attracting increasing attention, but how to increase its tumor-specificity remains a daunting challenge. Herein we report a theranostic probe (azo-PDT) that integrates pyropheophorbide $\alpha$ as a photosensitizer and a NIR fluorophore for tumor imaging. The two functionalities are linked with a hypoxic-sensitive azo group. Under normal conditions, both the phototoxicity of the photosensitizer and the fluorescence of the fluorophore are inhibited. While under hypoxic condition, the reductive cleavage of the azo group will restore both functions, leading to tumor specific fluorescence imaging and phototoxicity. The results showed that azo-PDT selectively images BEL-7402 cells under hypoxia, and simultaneously inhibits BEL-7402 cell proliferation after near-infrared irradiation under hypoxia, while little effect on BEL-7402 cell viability was observed under normoxia. These results confirm the feasibility of our design strategy to improve the tumortargeting ability of photodynamic therapy, and presents azo-PDT probe as a promising dual functional agent.

Cancer is one of the most common causes of death, and more and more therapeutic strategies against this fatal disease have emerged in the past few decades. Among these strategies, photodynamic therapy has attracted much attention $^{1}$. This therapy is based on singlet oxygen produced by photosensitizers under the irradiation with light of a specific wavelength to damage tumor tissues (Fig. 1a). Since the photo-damaging effect is induced by the interaction between a photosensitizer and light, tumor-specific therapy may be realized by focusing the light to the tumor site. Therefore, this therapeutic strategy is supposed to harm healthy tissue less than traditional cytotoxic drugs. Photofrin, the first photodynamic therapy drug approved by the FDA, has been routinely used for the treatment of certain cancers, such as esophageal cancer, lung cancer, bladder cancer, cervical cancer, and skin cancer ${ }^{2}$. However, since Photofrin is always ready to undergo photochemical reactions to produce singlet oxygen in the presence of light $(630 \mathrm{~nm})$ and also exhibits a long tissue retention time, it may cause long-lasting cutaneous photosensitivity ${ }^{3}$. Therefore, patients who have been treated with Photofrin have to avoid sunlight for several weeks, which presents a universal limit for "always on" photosensitizers.

To improve the tumor specificity, two strategies are generally utilized ${ }^{1}$. The first one is the conjugation of photosensitizers to nanocarriers with tumor-targeted, controlled-release properties. While this strategy has shown promise in preclinical models, it is limited by a complicated formulation process. The second strategy is based on prodrug-like photosensitizers whose photoreactivity can only be activated in tumor-specific environments. Tumor tissues usually demonstrate typical microenvironments that are significantly different from

${ }^{1}$ College of Pharmaceutical Sciences, Zhejiang University, Hangzhou 310058, China. ${ }^{2}$ Collaborative Innovation Centre of Yangtze River Delta Region Green Pharmaceuticals, Zhejiang University of Technology, Hangzhou 310014, China. ${ }^{\circledR}$ email: Shengr@zju.edu.cn; lixin81@zju.edu.cn 
a) Previous work (always on photosensitizer)

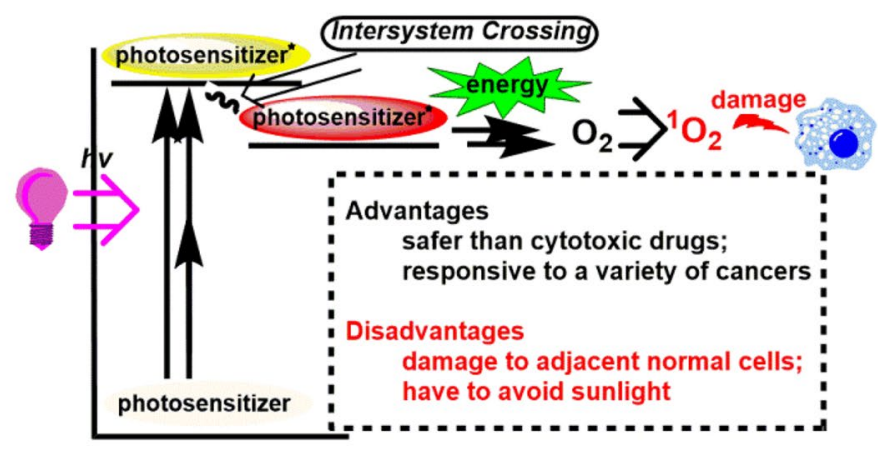

b)Our work:microenvironment-activatable tumor theranostic probe
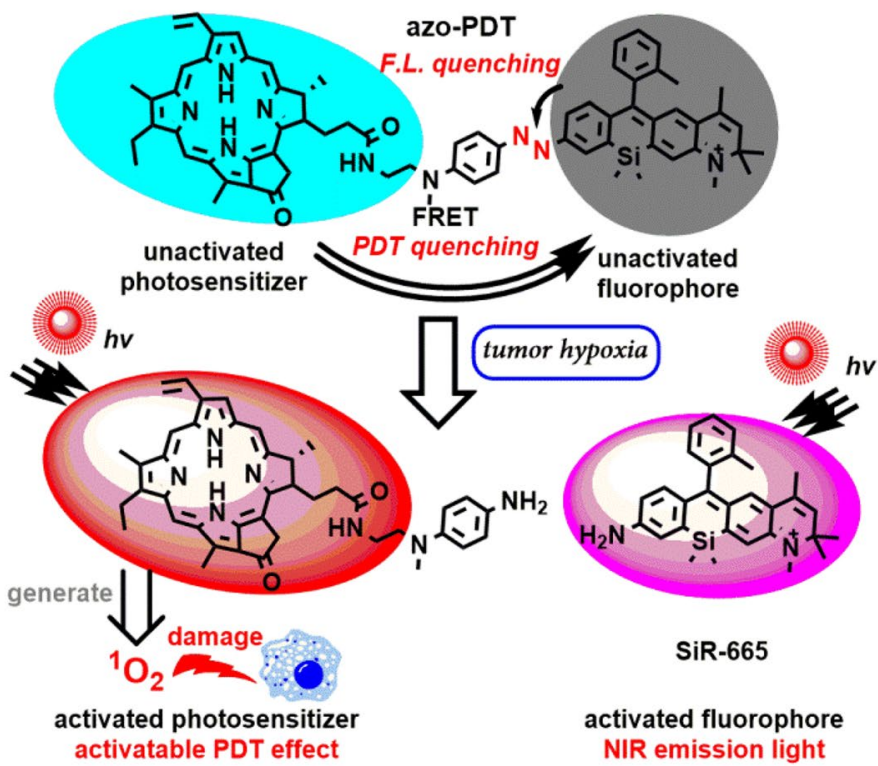

Figure 1. (a) Brief mechanism for ${ }^{1} \mathrm{O}_{2}$ generation in photodynamic therapy; (b) brief activation mechanism of azo-PDT under tumour hypoxia situations.

those of normal tissues. The features of these tumor microenvironments have been widely used for the design of tumor-targeted therapy ${ }^{4,5}$. Hypoxia is an important microenvironmental factor in cancer ${ }^{6}$. Therapies that are originally inert but sensitive to hypoxia may provide tumor-specificity. Taking advantage of hypoxia in solid tumors, many efforts have been devoted to develop tumor-targeted therapeutic or imaging agents ${ }^{7-16}$. Nagano and Urano et al. first reported that the azo group is sensitive toward hypoxia and may be employed to design fluorogenic probes for the detection of hypoxia ${ }^{17}$. Furthermore, they used the azo group as a quencher to design hypoxia-activated photosensitizers ${ }^{12}$. Recently, the groups of Tan, Fang, and Zhao collaborated to successfully design a hypoxia-activated aptamer for cancer imaging with improved specificity ${ }^{13}$. Based on hypoxia, Pu et al. successfully activated a prodrug of the chemotherapeutic drug Br-IPM ${ }^{14}$. All these results prove the advantage of the hypoxic tumor environment as a target to design tumor-specific imaging or therapy modalities. While many studies have been reported on hypoxia-activated therapeutic or imaging agents, hypoxia-dependent dual activation for simultaneous tumor imaging and photodynamic therapy has not been investigated to date, to the best of our knowledge.

To design bifunctional probes for hypoxia-dependent tumor imaging and photodynamic therapy, we started by interrogating the mechanism by which photosensitizers convert light energy into singlet oxygen. When a chromophore absorbs a photon, it is promoted to an excited state. The excited chromophore can lose energy by populating the first excited singlet state via internal conversion followed by rapid relaxation back to the ground state. However, for a photosensitizer, the excited singlet state electron is easily undergoing spin inversion to populate the first excited triplet state at lower energies via intersystem crossing. This triplet state readily interacts with ground-state molecular oxygen $\left({ }^{3} \mathrm{O}_{2}\right)$, which is a triplet state, leading to the production of radicals and reactive oxygen species ${ }^{1}$. Obviously, photochemical processes catalyzed by photosensitizers rely on the population of their triplet state. We hypothesized that inhibition of this state with a hypoxia-sensitive trigger may block the photochemical reaction under normoxia. Under hypoxia, the hypoxia-sensitive trigger switches on the photosensitizers and makes them ready for the photochemical reaction, leading to a hypoxia-specific photodynamic effect. 

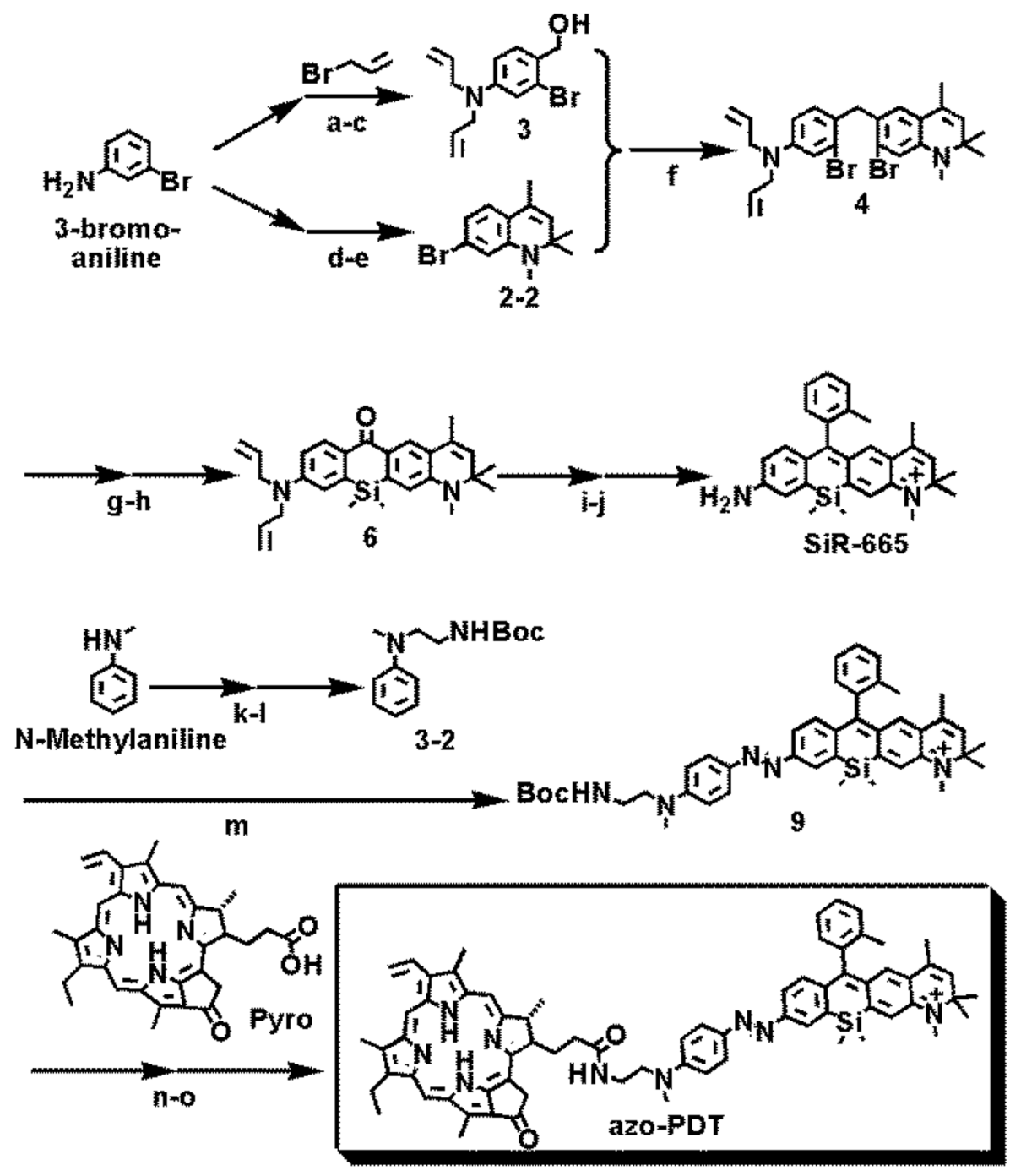

Figure 2. Synthesis of azo-PDT. Detailed reaction procedures were described in Scheme S1.

Fluorescence resonance energy transfer (FRET) is the absorption of the energy of a donor in its excited state by an acceptor structure, which may be employed to absorb the energy of an excited photosensitizer to inhibit the population in its excited triplet state. Herein, utilizing the FRET mechanism, we conjugated a photosensitizer (as energy donor) and a near-infrared fluorophore (as energy acceptor) with an azo group as hypoxia trigger, to successfully design a new chemical entity (azo-PDT) that can not only detect solid tumors based on its hypoxiaactivated fluorogenic response but also show hypoxia-specific phototoxicity for the ablation of tumor cells.

\section{Results and discussion}

Design and synthesis. We chose pyropheophorbide a (Pyro), which is an analog of Photofrin, as the photosensitizer for our proof-of-concept study due to its easy availability. For efficient FRET, donor emission and acceptor absorption should be adjacent and overlap ${ }^{18}$. Given the extremely low emission of Pyro around 700-800 nm, we reasoned that the Si-rhodamine fluorescent dye SiR-665 could act as the energy acceptor due to its absorption around $600-750 \mathrm{~nm}^{19}$. Another advantage of SiR-665 is its strong NIR II emission, which qualifies it as a fluorophore for tumor imaging. We designed the entity azo-PDT, which uses the azo group as hypoxia-specific trigger and also as linker between the photosensitizer Pyro and the fluorophore SiR-665. We reasoned that due to the FRET effect between Pyro and SiR-665, no photodynamic effect should be observed, not even under irradiation. Due to the azo-caused fluorescence quenching of SiR-665 ${ }^{\mathbf{1 7}}$, the probe should be non-emissive. Hypoxia will lead to the reductive cleavage of the azo group ${ }^{17}$, effectively separating the two functional moieties and, thus, activating both activity (Fig. 1b).

Procedures for the synthesis of azo-PDT are outlined in Fig. 2 and detailed in Scheme S1. Briefly, starting with 3-bromoaniline, we obtained comp.3 and comp.2-2. Friedel-Crafts reaction between comp.3 and comp.2-2 yielded the intermediate comp.4, which was further converted into SiR-665 following a reported procedures ${ }^{19}$. Diazo reaction of SiR-665 with comp.3-2 gave the azobenzene intermediate comp.9, and coupling of comp.9 


\begin{tabular}{|l|l|l|l|l|}
\hline Compound & $\lambda_{\text {abs }}(\mathbf{n m})$ & $\lambda_{\text {ex }}(\mathbf{n m})$ & $\lambda_{\text {em }}(\mathbf{n m})$ & $\Phi_{\Delta}{ }^{\mathrm{c}}$ \\
\hline Azo-PDT & $674^{\mathrm{a}}, 668^{\mathrm{b}}$ & $/$ & $/$ & $0.04^{\mathrm{b}}$ \\
\hline Methylene blue & $654^{\mathrm{b}}$ & - & - & $0.49^{\mathrm{b}}$ \\
\hline SiR-665 & $664^{\mathrm{a}}$ & $665^{\mathrm{a}}$ & $693^{\mathrm{a}}$ & - \\
\hline Pyro & $667^{\mathrm{b}}$ & $/$ & $/$ & $0.63^{\mathrm{b}}$ \\
\hline
\end{tabular}

Table 1. Photophysical and photochemical parameters of azo-PDT, methylene blue, SiR-665 and pyro. ${ }^{\mathrm{a}}$ The data were obtained in PBS. ${ }^{\mathrm{b}}$ The data were measured in EtOH. ${ }^{\mathrm{c}} \Phi_{\Delta}$ : singlet oxygen quantum yield. For methylene blue, $\Phi_{\Delta}=0.49$. "/" means not detectable, and “_" means not detected.

with Pyro yielded the desired compound azo-PDT (Fig. 2). The structures of azo-PDT and all precursors were characterized by NMR and mass spectrometry.

Characterization of the photophysical properties of azo-PDT. We studied the photophysical properties of azo-PDT by first testing if its photoreactivity and fluorescence were quenched. For this purpose, we first measured the absorption and emission properties of azo-PDT in comparison with those of SiR-665 (Table 1). Then, the singlet oxygen quantum yield $\left(\Phi\left({ }^{1} \mathrm{O}_{2}\right)\right)$ of azo-PDT was quantitatively measured in comparison with that of Pyro according to a reported method ${ }^{20}$. 1,3-Diphenylisobenzofuran (DPBF) was used as the ${ }^{1} \mathrm{O}_{2}$-capture and detection agent, and an EtOH solution of azo-PDT or Pyro in the presence of DPBF was irradiated at $670 \mathrm{~nm}$. Once ${ }^{1} \mathrm{O}_{2}$ is photo-induced, it would react with DPBF via 1,4-cycloaddition, resulting in a decrease of its absorbance at $410 \mathrm{~nm}$ (Fig. 3a). Using methylene blue $\left(\Phi\left({ }^{1} \mathrm{O}_{2}\right)=0.49\right)$ as a standard ${ }^{21}$, the $\Phi\left({ }^{1} \mathrm{O}_{2}\right)$ values of azo-PDT and Pyro were calculated as 0.04 and 0.63, respectively (Table 1 ). The significantly lower $\Phi\left({ }^{1} \mathrm{O}_{2}\right)$ value of azo-PDT compared with Pyro suggests effective quenching of the photoreactivity of azo-PDT due to the efficient FRET process between Pyro and SiR-665. This is in accord with the photophysical data, which showed partial overlap between the emission of Pyro and the absorption of SiR-665 (Fig. 3b).

After confirming the effective quenching of the ${ }^{1} \mathbf{O}_{2}$-production ability of azo-PDT, we measured its fluorescence properties. As shown in Fig. 3b, azo-PDT is almost non-fluorescent, although SiR-665 is highly emissive. This finding indicates that the fluorescence of SiR-665 fluorophore is significantly quenched by the azo group.

Hypoxia-activated fluorescence of azo-PDT in aqueous solution. After confirming the effective inhibition of the ${ }^{1} \mathrm{O}_{2}$-generation ability of azo-PDT and quenching of its fluorescence emission, we tested if reductive cleavage of the azo group restores both activities. For this purpose, the biological reductive hypoxic environment was mimicked with mouse liver microsomes ${ }^{17}$. After treating azo-PDT $(5 \mu \mathrm{M})$ with mouse liver microsomes $(75 \mu \mathrm{g} / \mathrm{mL})$ in the presence of NADPH $(50 \mu \mathrm{M})$ as a reductase cofactor, an increase of the probe fluorescence was observed, which intensified with the proceeding of the incubation (Fig. $4 \mathrm{a}$ ).

We also checked if other biological species commonly found in live cells induce the switch-on of the fluorescence of azo-PDT. For this purpose, azo-PDT in PBS was treated with various analytes for $1 \mathrm{~h}$, and then its fluorescence was observed. As shown in Fig. 4b, microsomes were the only analytes to induce its fluorescence, suggesting that azo-PDT is specifically activated by the biological hypoxic environment. This is highly advantageous, as this suggests that azo-PDT should have a tumor-specific photodynamic effect.

Furthermore, we also confirmed the reductive cleavage of the azo bond in azo-PDT to yield the free aminoSiR-665 by LC-MS analysis (Fig. S1), supporting our design rationale.

Hypoxia-activated fluorescence and photoreactivity of azo-PDT in live cells. Having confirmed the hypoxia-activated fluorescence of azo-PDT in aqueous solution, we studied if azo-PDT retains this feature in live cells for hypoxia-specific imaging and photodynamic ablation. For this purpose, we first screened a panel of cells to select the most sensitive one and applied azo-PDT to these cells under hypoxia $\left(1 \% \mathrm{O}_{2}\right)$. All cells demonstrated higher intracellular probe fluorescence under hypoxia than under normoxia (Fig. S2), suggesting that azo-PDT may be activated under hypoxia in all tested cell lines. It is noteworthy that BEL-7402 cells showed the most dramatic intracellular fluorescence increase under hypoxia, suggesting that azo-PDT is more sensitive in BEL-7402 cells, which were thus subject of further research.

First, the influence of the hypoxia incubation time on the activation of azo-PDT was studied by detecting the fluorescence switch-on effect. For this purpose, BEL-7402 cells were incubated with azo-PDT $(5 \mu \mathrm{M})$ under hypoxia or normoxia for various times. While no significant time-dependent increase of the intracellular fluorescence was observed in the normoxia group, the intracellular azo-PDT fluorescence increased with increasing incubation time in the hypoxia group. These results suggest that, under hypoxia, the azo group in azo-PDT is reductively cleaved, which occurs gradually with increasing incubation times (Fig. 5).

In the following, we optimized the working concentration of azo-PDT to stain cells. For this purpose, BEL7402 cells were incubated with various concentrations of azo-PDT under normoxia or hypoxia for $6 \mathrm{~h}$. Cells under normoxia demonstrated negligible intracellular azo-PDT fluorescence, indicating that the fluorescence of azo-PDT is quenched, while the intracellular fluorescence of cells under hypoxia depended on the azo-PDT concentration (Fig. S3). Our results showed that an azo-PDT concentration of $2.5 \mu \mathrm{M}$ was sufficient to yield significant intracellular fluorescence under hypoxia. Therefore, we chose this azo-PDT working concentration 
a)
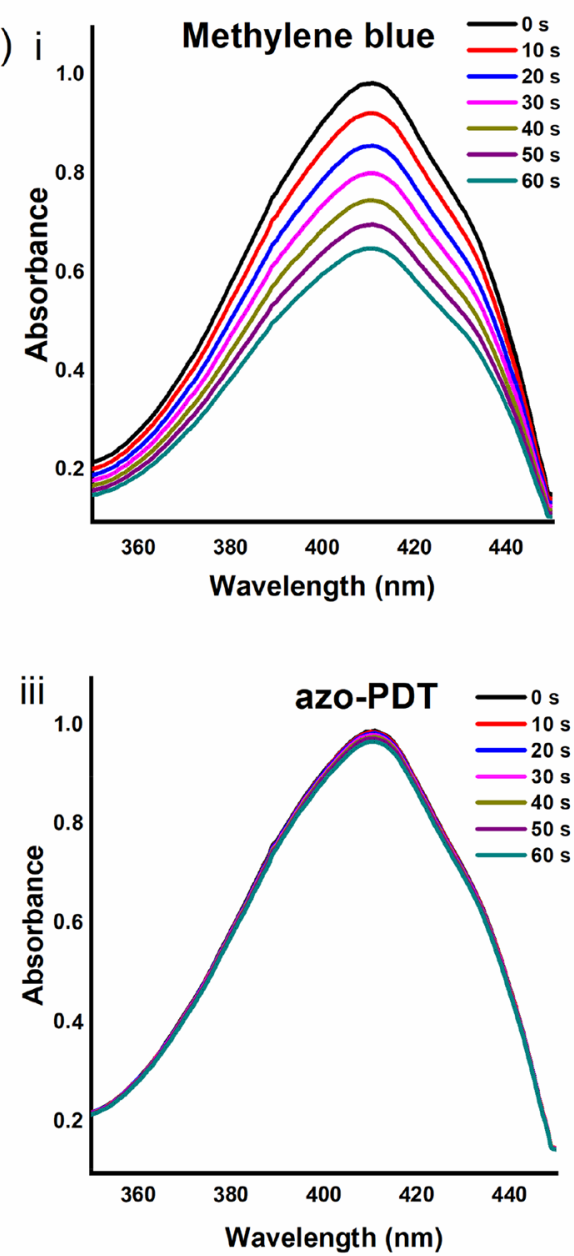

b)

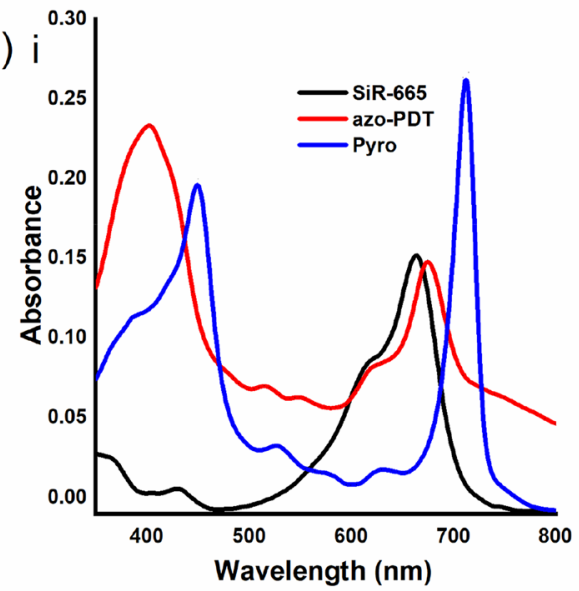

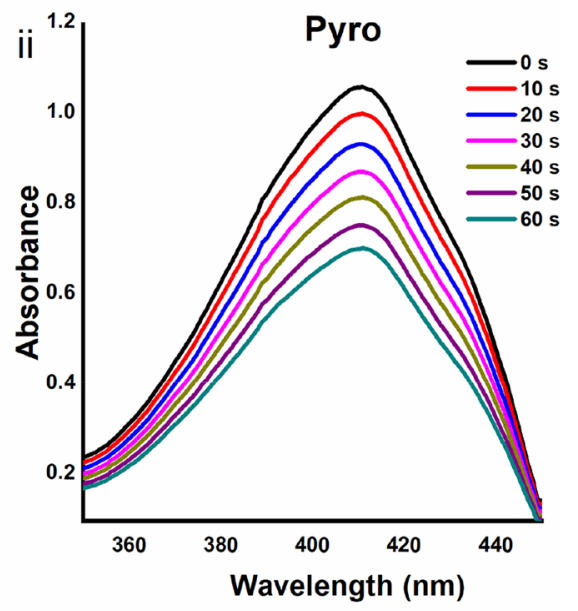
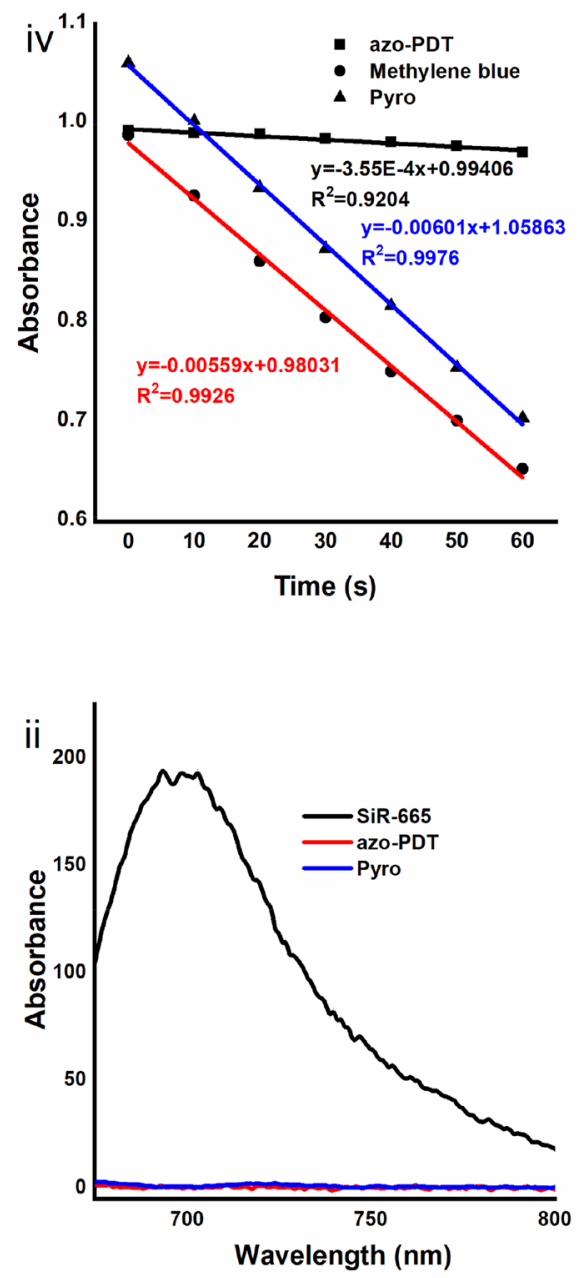

Figure 3. (a) (i-iii) Change of DPBF absorption spectra after photo-irradiation of the photosensitizers.

Methylene blue (i), Pyro (ii) or azo-PDT (iii) was dissolved at $1 \mu \mathrm{M}$ in EtOH. DPBF was added to the solution to a final concentration of $40 \mu \mathrm{M}$. The solution was irradiated with light filtered around the maximum absorption wavelength $(670 \mathrm{~nm})$. The absorption spectrum was obtained every $10 \mathrm{~s}$. (iv) Decay curves of the $410 \mathrm{~nm}$ absorption bands in i, ii, and iii. The power of LED lamp was $150 \mathrm{~W}$ and the distance of irradiation was $80 \mathrm{~cm}$; (b) Absorption (i) and fluorescent (ii) spectra of $5 \mu \mathrm{M}$ of SiR-665, azo-PDT and Pyro in PBS. 

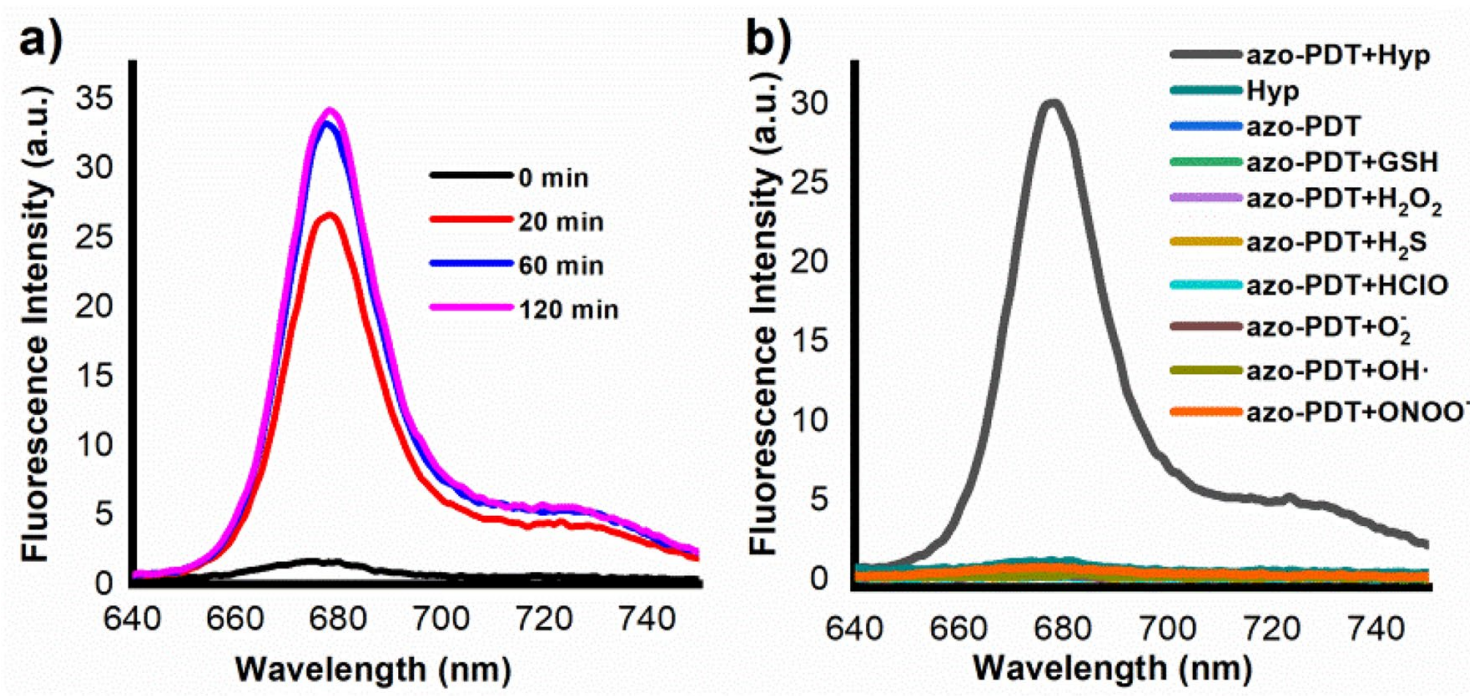

Figure 4. (a) Time-dependent changes in the fluorescence intensity of azo-PDT ( $5 \mu \mathrm{M})$ in the presence of mouse liver microsomes $(226 \mu \mathrm{g} / 3 \mathrm{~mL})$; (b) fluorescent responses of azo-PDT ( $5 \mu \mathrm{M})$ towards various analytes. Data shown were the fluorescence intensity of the probe at $680 \mathrm{~nm}$ after being incubated with various analytes for $60 \mathrm{~min}$. All fluorescence data were collected in PBS (pH 7.4, $100 \mathrm{mM}$ ) containing 5\% DMF as co-solvent at $37^{\circ} \mathrm{C}$ with $\lambda_{\text {ex }} 420 \mathrm{~nm}$.

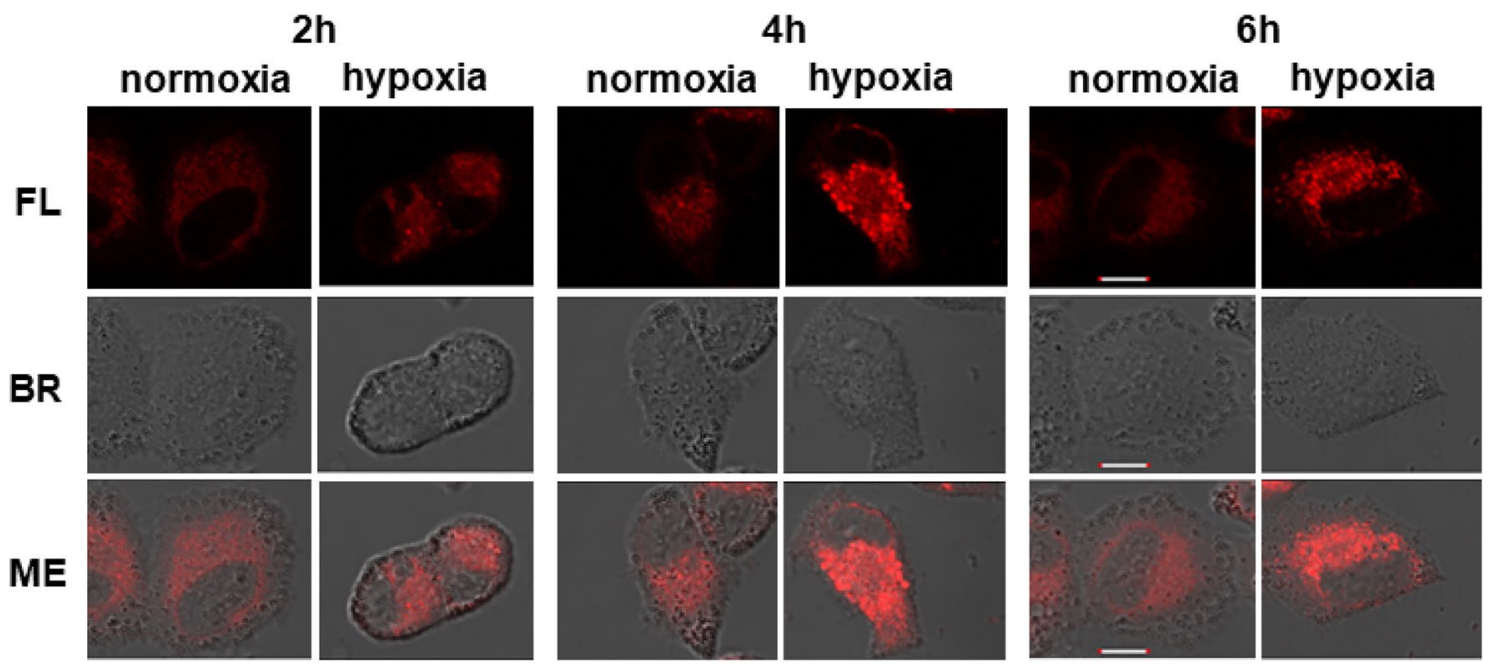

Figure 5. Time-dependent change of the fluorescence intensity from BEL-7402 cells with $5 \mu \mathrm{M}$ azo-PDT containing DMSO $(0.1 \%)$ and RH40 (0.1\%) as cosolvent. Scale bar: $10 \mu \mathrm{m}$. FL fluorescence image, BR bright field image. The excitation and emission wavelengths were $640 \mathrm{~nm}$ and $650-750 \mathrm{~nm}$, respectively.

and an incubation time of $6 \mathrm{~h}$ for the following cell experiments. Under these conditions, the intracellular fluorescence intensity of azo-PDT was 1.8-fold higher under hypoxia than under normoxia (Fig. S4).

After confirming the hypoxia-dependent activation of the fluorescence of azo-PDT in BEL-7402 cells, we tested if hypoxia also restores the ${ }^{1} \mathrm{O}_{2}$-generation ability of azo-PDT in BEL-7402 cells. BEL-7402 cells were incubated with azo-PDT under normoxia or hypoxia for $6 \mathrm{~h}$ and then irradiated with LED light at $670 \mathrm{~nm}$ for $20 \mathrm{~min}$ to induce the production of ${ }^{1} \mathrm{O}_{2}$. Then, the cells were incubated without irradiation for another $24 \mathrm{~h}$, followed by SRB assay and cck-8 assay to measure the cell viability using Pyro as a positive control. While the Pyro group showed an irradiation-dependent cell ablation effect under both normoxia and hypoxia, low concentrations of azo-PDT only ablated the cell viability under hypoxia after irradiation, suggesting its hypoxia specificity (Fig. S5). The cytotoxicity of azo-PDT $(2.5 \mu \mathrm{M})$ under normoxia or hypoxia, and with or without irridaition was summarized in Fig. 6 with pyro as a positive control. In contrast to pyro which shows photoirradiation-dependent cytotoxicity either under normoxia or hypoxia, azo-PDT showed potent cytotoxicity only under hypoxia when photo-irradiated. This observation suggests that the cell ablation effect of azo-PDT relies on both photo-irradiation and hypoxia activation, which confirms the success of our designed probe. To make further confirmation that the hypoxia-photo-irradiation-dependent cytotoxicity of azo-PDT is indeed due to its induction of ROS generation, we checked the cellular ROS levels by staining cells with $2^{\prime}, 7^{\prime}$-dichlorofluorescin 


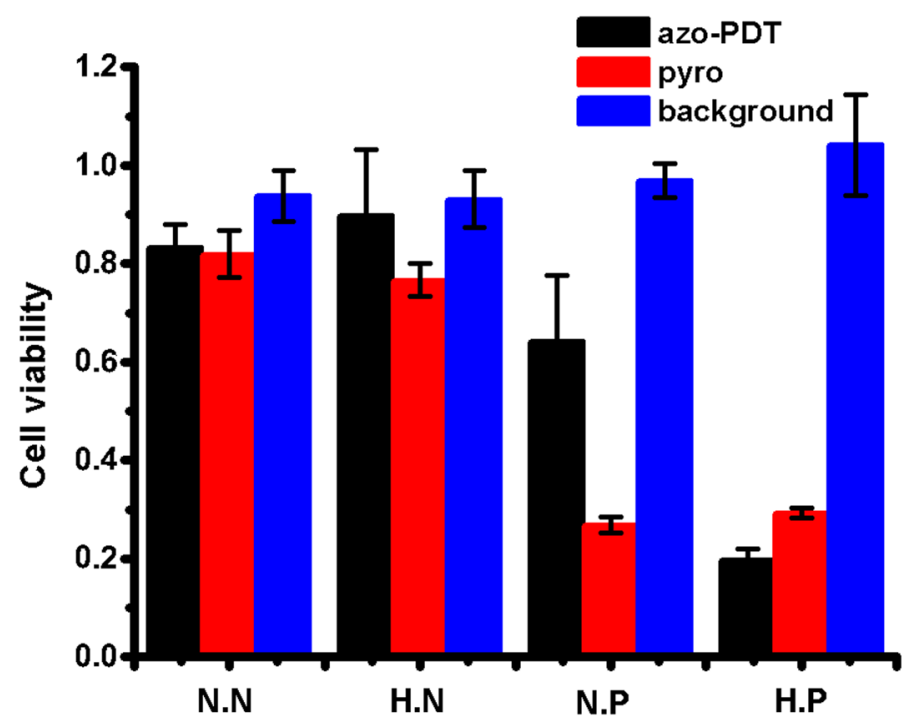

Figure 6. Survival viabilities of BEL-7402 cells after the treatment of azo-PDT or Pyro at $2.5 \mu \mathrm{M}$ under normoxia or hypoxia, followed with or without photoirradiation. SRB assay was used to monitor cell viabilities. N.N normoxia with no photoirradiation, H.N hypoxia with no photoirradiation, N.P normoxia with photoirradiation, H.P hypoxia with photoirradiation. The power of LED lamp was $150 \mathrm{~W}$ and the distance of irradiation was $6.5 \mathrm{~cm}$.

diacetate, a ROS indicator. BEL-7402 cells were incubated with azo-PDT $(2.5 \mu \mathrm{M})$ under normoxia or hypoxia for $6 \mathrm{~h}$. Cells in the hypoxia group were then irradiated with LED light at $670 \mathrm{~nm}$ for $20 \mathrm{~min}$, while cells in the normoxia group were kept under normal indoor light. Cells in all groups were then stained with $2^{\prime}$, $7^{\prime}$-dichlorofluorescin diacetate $(5 \mu \mathrm{M})$ for $15 \mathrm{~min}$, and then imaged under microscopy. As shown in Fig. S6, only cells in the hypoxia and irradiated group showed bright fluorescein fluorescence, indicating the upregulation of cellular ROS in this group. This result suggests that activated azo-PDT can still induce sufficient ROS even under hypoxia.

In summary, utilizing the resonant energy transfer between pyropheophorbide $\alpha$ and the quenched fluorophore SiR-665, we have developed a "pro-photosensitizer" that is activated under hypoxia in tumor cells. Due to the energy transfer between the photosensitizer and the quenched fluorophore, the pro-photosensitizer does not generate singlet oxygen to damage cells under normoxia. Under hypoxia, the azo group undergoes reductive cleavage, effectively separating the photosensitizer and fluorophore and, consequently disrupting the energy transfer between these groups and restoring the fluorescence of the fluorophore as well as the photoactivity of the photosensitizer. Applying this strategy, tumor-selective imaging and photodynamic therapy may be realized. We validated the feasibility of this strategy in live BEL-7402 cells. We have shown that $2.5 \mu \mathrm{M}$ azo-PDT achieved real-time imaging and showed selective killing activity. Overall, azo-PDT may be a promising dual-functional imaging tool to detect cancer hypoxia as well as to achieve tumor-specific cancer therapy. Furthermore, the design strategy introduced in this study may be inspiring for future probe design.

\section{Methods}

Detailed synthetic routes were described in supplementary information.

Photophysical and photochemical property measurements. Deionized water was used to prepare all aqueous solutions. The concentration of phosphate buffer saline (PBS, pH 7.4) was 0.1 M. Dyes (Pyro, SiR665 or azo-PDT) were dissolved in dimethyl sulfoxide (DMSO) to make stock solutions. The UV absorption spectra were measured with a HITACHI U-3010 Spectrophotometer. The fluorescence measurements were obtained on an Agilent Cary Eclipse Fluorescence Spectrophotometer. The excitation and emission slit widths were $5 \mathrm{~nm}$.

Singlet oxygen detection by 1,3-diphenylisobenzofuran (DPBF). Photosensitizers (Pyro, azoPDT or methylene blue) with DPBF $(40 \mu \mathrm{M})$ were dissolved in ethanol (EtOH). The solution was irradiated with a HollandStar GL-150 W LED light $(670 \mathrm{~nm})$. The rate of DPBF consumption (i.e., the slope) which corresponded to the relative efficiency of ${ }^{1} \mathrm{O}_{2}$ generation by the irradiated photosensitizer was calculated according to the relative number of absorbed photons around the maximum absorbance of each dye ${ }^{22}$. Methylene blue $\left(\Phi\left({ }^{1} \mathrm{O}_{2}\right)=0.49\right.$ in EtOH $)$ was used as a standard ${ }^{23}$. The ${ }^{1} \mathrm{O}_{2}$ quantum yield was calculated by comparison with a standard photosensitizer, $\Phi=\Phi_{s}{ }^{*} k^{\star} I_{s} /\left(k_{s}{ }^{*}\right)$, where $\mathrm{k}$ and $\mathrm{k}_{\mathrm{s}}$ were the rate constants for decomposition of DPBF by Pyro or azo-PDT and by methylene blue as a standard photosensitizer, respectively. I and $\mathrm{I}_{\mathrm{s}}$ represented light absorbed by Pyro or azo-PDT and by methylene blue, respectively, which were determined by the wavelength of light source ${ }^{20}$. 
In vitro fluorescent assay with mouse liver microsomes. The enzyme assay in cuvette (hypoxic condition in vitro) was prepared by bubbling $\mathrm{N}_{2}$ into the $0.1 \mathrm{M}$ PBS ( $\mathrm{pH}$ 7.4) for at least 30 min. Mouse liver microsomes $(226 \mu \mathrm{g} / 3 \mathrm{~mL}$ ) from Research Institute for Liver Diseases (Shanghai) Co. Ltd and $5 \mu \mathrm{M}$ azo-PDT was added into the reaction solution containing $5 \% \mathrm{DMF}$ as a cosolvent. Then $50 \mu \mathrm{M}$ NADPH as a cofactor for reductases was added.

Cell lines and culture conditions. Human hepatoma cells BEL-7402 were supplied by collaborators. BEL7402, KM12, A549 and DU145 cells were cultured in RPMI 1640 medium (Invitrogen) supplemented with $10 \%$ heat-inactivated FBS (Gibico). HepG2 and MCF7 cells were cultured in DMEM medium (Invitrogen) supplemented with $10 \%$ heat-inactivated FBS (Gibico). B16F10 cells were cultured in DMEM/F-12 (1/1) medium (Invitrogen) supplemented with $10 \%$ heat-inactivated FBS (Gibico). All cell lines were incubated at $37^{\circ} \mathrm{C}$ under an atmosphere of $5 \% \mathrm{CO}_{2}$ in air.

Hypoxic condition for live cells fluorescence imaging. An $\mathrm{O}_{2}$ concentration of $1 \%$ was controlled by the Thermo Scientific Forma $\mathrm{CO}_{2}$ incubator by means of $\mathrm{N}_{2}$ substitution. Confocal Fluorescence images were obtained on Olympus IX83-FV3000. The fluorescence density was calculated using Image J software (NIH, Bethesda, MD, USA).

Cell culture. Different cell lines. $1.5 \times 10^{5}$ cells were seeded onto $3.5 \mathrm{~cm}$ Petri dishes and cultured in corresponding medium with $10 \%$ (v/v) FBS and incubated for $12 \mathrm{~h}$. The tested compounds were diluted to $5 \mu \mathrm{M}$, then the cells were incubated for $1 \mathrm{~h}$ under an atmosphere of $5 \% \mathrm{CO}_{2}$ in air, thereafter the cells were incubated for $6 \mathrm{~h}$ under an atmosphere of $5 \% \mathrm{CO}_{2}$ and $1 \% \mathrm{O}_{2}$ (hypoxia) or for $6 \mathrm{~h}$ under an atmosphere of $5 \% \mathrm{CO}_{2}$ in air (normoxia). The medium was removed and washed with fresh medium. Confocal Fluorescence images were then obtained on Olympus IX83-FV3000.

Different hypoxia hours. $1.5 \times 10^{5}$ cells were seeded onto $3.5 \mathrm{~cm}$ Petri dishes and cultured in RPMI 1640 medium with $10 \%(\mathrm{v} / \mathrm{v}) \mathrm{FBS}$ and incubated for $12 \mathrm{~h}$. The tested compounds were diluted to $5 \mu \mathrm{M}$, then the cells were incubated for $1 \mathrm{~h}$ under an atmosphere of $5 \% \mathrm{CO}_{2}$ in air, thereafter the cells were incubated for different hours under an atmosphere of $5 \% \mathrm{CO}_{2}$ and $1 \% \mathrm{O}_{2}$. The medium was removed and washed with fresh medium. Confocal Fluorescence images were then obtained on Olympus IX83-FV3000.

Different concentrations. $1.5 \times 10^{5}$ cells were seeded onto $3.5 \mathrm{~cm}$ Petri dishes and cultured in RPMI 1640 medium with $10 \%(\mathrm{v} / \mathrm{v})$ FBS and incubated for $12 \mathrm{~h}$. The tested compounds were diluted to different final concentrations, then the cells were incubated for $1 \mathrm{~h}$ under an atmosphere of $5 \% \mathrm{CO}_{2}$ in air, thereafter the cells were incubated for $6 \mathrm{~h}$ under an atmosphere of $5 \% \mathrm{CO}_{2}$ and $1 \% \mathrm{O}_{2}$ (hypoxia) or for $6 \mathrm{~h}$ under an atmosphere of $5 \% \mathrm{CO}_{2}$ in air (normoxia). The medium was removed and washed with fresh medium. Confocal Fluorescence images were then obtained on Olympus IX83-FV3000.

Hypoxia-triggered photodynamic ablation of tumor cells. Different concentrations. $5 \times 10^{3}$ cells were seeded on 96-well plates and cultured in RPMI 1640 medium with 10\% (v/v) FBS for $24 \mathrm{~h}$. The tested compounds were diluted to different final concentrations, then the cells were incubated for $1 \mathrm{~h}$ under an atmosphere of $5 \% \mathrm{CO}_{2}$ in air, thereafter the cells were incubated for $6 \mathrm{~h}$ under an atmosphere of $5 \% \mathrm{CO}_{2}$ and $1 \% \mathrm{O}_{2}$ (hypoxia) or for $6 \mathrm{~h}$ under an atmosphere of $5 \% \mathrm{CO}_{2}$ in air (normoxia). Then the plate was irradiated by LED light $(670 \mathrm{~nm})$ for $20 \mathrm{~min}$ and cells were incubated under normal atmosphere for another $24 \mathrm{~h}$. Cell viability was then measured using SRB assay or cck-8 assay.

Received: 23 February 2020; Accepted: 22 June 2020

Published online: 22 July 2020

\section{References}

1. Chilakamarthi, U. \& Giribabu, L. Photodynamic therapy: Past, present and future. Chem. Rec. 17, 775-802 (2017).

2. Dougherty, T. J., Grindey, G. B., Fiel, R., Weishaupt, K. R. \& Boyle, D. G. Photoradiation therapy. II. Cure of animal tumors with hematoporphyrin and light. J. Natl. Cancer. Inst. 55, 115-121 (1975).

3. Dougherty, T. J., Cooper, M. T. \& Mang, T. S. Cutaneous phototoxic occurrences in patients receiving Photofrin. Lasers Surg. Med. 10, 485-488 (1990).

4. Najafi, M. et al. Tumor microenvironment: Interactions and therapy. J. Cell. Physiol. 234, 5700-5721 (2019).

5. Zeng, L. et al. A GSH-activatable ruthenium(ii)-azo photosensitizer for two-photon photodynamic therapy. Chem. Commun. 53, 1977-1980 (2017).

6. Brown, J. M. Tumor hypoxia in cancer therapy. Method. Enzymol. 435, 295-321 (2007).

7. Li, Y. et al. Ultrasensitive near-infrared fluorescence-enhanced probe for in vivo nitroreductase imaging. J. Am. Chem. Soc. 137, 6407-6416 (2015)

8. Silvers, W. C., Payne, A. S. \& McCarley, R. L. Shedding light by cancer redox-human NAD(P)H: Quinone oxidoreductase 1 activation of a cloaked fluorescent dye. Chem. Commun. 47, 11264-11266 (2011).

9. Gontijo, T. B. et al. Novel fluorescent lapachone-based BODIPY: Synthesis, computational and electrochemical aspects, and subcellular localisation of a potent antitumour hybrid quinone. Chem. Commun. 52, 13281-13284 (2016).

10. Xu, K. et al. High selectivity imaging of nitroreductase using a near-infrared fluorescence probe in hypoxic tumor. Chem. Commun. 49, 2554-2556 (2013). 
11. Piao, W. et al. Development of azo-based fluorescent probes to detect different levels of hypoxia. Angew. Chem. Int. Ed. 52, 13028-13032 (2013).

12. Piao, W. et al. Development of an azo-based photosensitizer activated under mild hypoxia for photodynamic therapy. J. Am. Chem. Soc. 139, 13713-13719 (2017).

13. Zhou, F. et al. Hypoxia-activated PEGylated conditional aptamer/antibody for cancer imaging with improved specificity. J. Am. Chem. Soc. 141, 18421-18427 (2019).

14. Cui, D. et al. A semiconducting polymer nano-prodrug for hypoxia-activated photodynamic cancer therapy. Angew. Chem. Int. Ed. 58, 5920-5924 (2019).

15. Huang, J., Wu, Y., Zeng, F. \& Wu, S. An activatable near-infrared chromophore for multispectral optoacoustic imaging of tumor hypoxia and for tumor inhibition. Theranostics. 9, 7313-7324 (2019).

16. Ding, N. et al. Azo-based near-infrared fluorescent theranostic probe for tracking hypoxia-activated cancer chemotherapy in vivo. Chem. Commun. 55, 13172-13175 (2019).

17. Kiyose, K. et al. Hypoxia-sensitive fluorescent probes for in vivo real-time fluorescence imaging of acute ischemia. J. Am. Chem. Soc. 132, 15846-15848 (2010).

18. Jares-Erijman, E. \& Jovin, T. FRET imaging. Nat. Biotechnol. 21, 1387-1395 (2003).

19. Koide, Y. et al. Development of NIR fluorescent dyes based on si-rhodamine for in vivo imaging. J. Am. Chem. Soc. 134, 5029-5031 (2012).

20. Xiao, L., Gu, L., Howell, S. B. \& Sailor, M. J. Porous silicon nanoparticle photosensitizers for singlet oxygen and their phototoxicity against cancer cells. ACS Nano 5, 3651-3659 (2011).

21. Redmond, R. W. \& Gamlin, J. N. A compilation of singlet oxygen yields from biologically relevant molecules. Photochem. Photobiol. 70, 391-475 (1999).

22. Venkatesan, R., Periasamy, N. \& Srivastava, T. S. Singlet molecular oxygen quantum yield measurements of some porphyrins and metalloporphyrins. J. Chem. Sci. 104, 713-722 (1992).

23. Redmond Robert, W. \& Gamlin Janet, N. A compilation of singlet oxygen yields from biologically relevant molecules. Photochem. Photobiol. 70, 391-475 (1999).

\section{Acknowledgements}

This study was financially supported by the National Natural Science Foundation of China (21672187), the Natural Science Foundation of Science and Technology Department of Zhejiang Province (LZ17H300002), and Key New Drug Creation and Manufacturing Program, China (2018ZX09711002-010-004).

\section{Author contributions}

C.W., S.W. and K.B. synthesize all the compounds; H.W. guide the biological experiments. C.W., Y.W. carry out biological evaluation. C.W. and X.L. write the manuscript, R.S. and X.L. designed all the experiments and revised the manuscript.

\section{Competing interests}

The authors declare no competing interests.

\section{Additional information}

Supplementary information is available for this paper at https://doi.org/10.1038/s41598-020-68847-w.

Correspondence and requests for materials should be addressed to R.S. or X.L.

Reprints and permissions information is available at www.nature.com/reprints.

Publisher's note Springer Nature remains neutral with regard to jurisdictional claims in published maps and institutional affiliations.

(c) (1) Open Access This article is licensed under a Creative Commons Attribution 4.0 International License, which permits use, sharing, adaptation, distribution and reproduction in any medium or format, as long as you give appropriate credit to the original author(s) and the source, provide a link to the Creative Commons license, and indicate if changes were made. The images or other third party material in this article are included in the article's Creative Commons license, unless indicated otherwise in a credit line to the material. If material is not included in the article's Creative Commons license and your intended use is not permitted by statutory regulation or exceeds the permitted use, you will need to obtain permission directly from the copyright holder. To view a copy of this license, visit http://creativecommons.org/licenses/by/4.0/.

(c) The Author(s) 2020 